\title{
Approaching the ultimate open circuit voltage in thiophene based single junction solar cells by applying diindenoperylene as acceptor
}

\author{
Ulrich Hörmann*, Julia Wagner, Mark Gruber, Andreas Opitz, and Wolfgang Brütting* \\ Institut für Physik, Universität Augsburg, 86135 Augsburg, Germany \\ Received XXXX, revised XXXX, accepted XXXX \\ Published online XXXX
}

Key words: diindenoperylene, sexithiophene, P3HT, organic photovoltaic cell

* Corresponding author: e-mail ulrich.hoermann@physik.uni-augsburg.de, Phone: +49-821-5983412, Fax: +49-821-5983425 e-mail wolfgang.bruetting@physik.uni-augsburg.de, Phone: +49-821-5983403, Fax: +49-821-5983425

The efficiency of a photovoltaic cell is directly proportional to its open circuit voltage. This in turn is eventually set by the donor-acceptor energy gap, i.e. the energy of the intermolecular charge-transfer state in organic solar cells. In this letter we study diindenoperylene (DIP) as a new molecular acceptor. We show that planar heterojunctions of thiophene derivatives and DIP yield extraordinarily high open circuit voltages of approximately $1.2 \mathrm{~V}$ for poly(3-hexylthiophene) and almost $1.4 \mathrm{~V}$ for heat treated $\alpha$-sexithiophene. Those values are close to the maximum $V_{\text {oc }}$ attainable for these material systems.

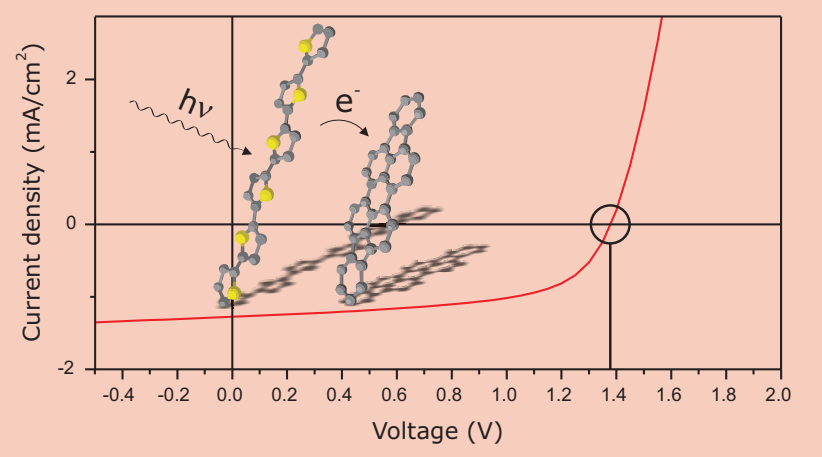

Copyright line will be provided by the publisher
The open circuit voltage $V_{\mathrm{oc}}$ of organic heterojunction solar cells has been the subject of intense research in recent years. Even though its origin is not yet completely understood, it is generally accepted that the donor-acceptor (D/A) intermolecular gap is decisive for the $V_{\mathrm{oc}}$ reached by solar cell devices [1-3]. This gap is determined by the relative energetic position of the highest occupied molecular orbital (HOMO) of the donor and the lowest unoccupied molecular orbital (LUMO) of the acceptor material. For a D/A system to be suitable for solar cell applications, the HOMO-HOMO and LUMO-LUMO offsets need to be sufficiently large to efficiently drive the exciton dissociation. However, if this energy offset is too big, a major fraction of the incoming photon energy is lost. As a rule of thumb, the ultimate limit of $V_{\mathrm{oc}}$ is considered to be about $0.5-0.6 \mathrm{eV}$ below the optical gap of the absorber material [2].
While a large variety of donor materials has been investigated in the literature, the choice of acceptors is dominated by the class of fullerenes, most prominently $\mathrm{C}_{60}$ and its soluble derivatives. Yet, the first organic heterojunction solar cell presented by Tang in 1986 comprises a perylene derivative as the electron accepting material [4]. Recently another perylene derivative, diindenoperylene (DIP), was introduced as a new donor in $\mathrm{DIP} / \mathrm{C}_{60}$ solar cells exhibiting power conversion efficiencies up to $4 \%$ [5]. Additionally ambipolar transport in DIP was found in transistor devices, where the electron mobility even outranges the hole mobility by a factor of two [6]. Good electron transport potentially enables DIP to also act as the acceptor in a solar cell, if a suitable donor can be found.

Figure 1 shows the energy levels of $\alpha$-sexithiophene (6T), DIP and $\mathrm{C}_{60}$. Besides the obvious combinations of 


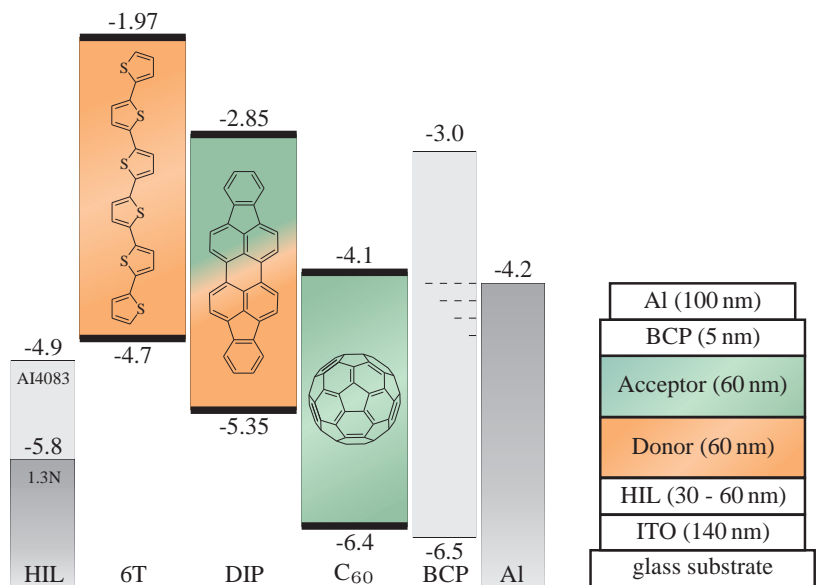

Figure 1 Schematic drawing of molecular formulae and energy levels (in eV) of 6T, DIP and $\mathrm{C}_{60}$. Energy values of organic materials are taken from $[5,7,8], 6 \mathrm{~T}$ LUMO is estimated by adding the transport gap [9] to the HOMO. Defect states enabling electron transport through BCP are sketched [8]. The layer stack of the investigated solar cell devices is shown on the right.

6T/ $\mathrm{C}_{60}$ and $\mathrm{DIP} / \mathrm{C}_{60}$ - both employing $\mathrm{C}_{60}$ as acceptor the relative energetic position of $6 \mathrm{~T}$ and DIP suggests a solar cell device in which DIP is used as the electron acceptor. From this figure the intermolecular gap of the different $\mathrm{D} / \mathrm{A}$ pairs is estimated to $0.6 \mathrm{eV}, 1.25 \mathrm{eV}$ and $1.85 \mathrm{eV}$ for $6 \mathrm{~T} / \mathrm{C}_{60}, \mathrm{DIP} / \mathrm{C}_{60}$ and 6T/DIP, respectively. The linear dependence of $V_{\mathrm{oc}}$ on the gap [2] implies an open circuit voltage that is approximately twice as high for $\mathrm{DIP} / \mathrm{C}_{60}$ and three times as high for 6T/DIP with respect to the $6 \mathrm{~T} / \mathrm{C}_{60}$ device.

To test this hypothesis planar heterojunction solar cells as illustrated in Figure 1 were fabricated. ITO covered glass slides were coated with a polymeric hole injection layer (HIL1.3N or AI4083, Heraeus Clevios) and dried on a hotplate $\left(150^{\circ} \mathrm{C}\right)$. Thereafter the samples were transferred to a high vacuum system $\left(2 \times 10^{-7} \mathrm{mbar}\right)$, where $60 \mathrm{~nm}$ of the donor and subsequently $60 \mathrm{~nm}$ of the acceptor were deposited. Finally a bathocuproine (BCP) buffer layer and the aluminum cathode were evaporated in an additional vacuum chamber $\left(2 \times 10^{-7} \mathrm{mbar}\right)$. Molecular materials were purchased from Sigma Aldrich (6T, BCP), University of Stuttgart (DIP), Creaphys $\left(\mathrm{C}_{60}\right)$ and - except for BCP - purified twice by temperature gradient sublimation. Because of its high work function the PEDOT formulation HIL1.3N was chosen as the hole injection layer to avoid the s-shape present in $\mathrm{DIP} / \mathrm{C}_{60}$ devices employing the standard PEDOT:PSS formulation AI4083 as reported in [5]. The ITO pattern and the cathode geometry yield an active area of $0.04 \mathrm{~cm}^{2}$. For characterization currentvoltage curves have been recorded under white light illumination using an LED (Luxeon LXHL-NWE8) as light source with a sharp emission peak at $453 \mathrm{~nm}$ and a broad

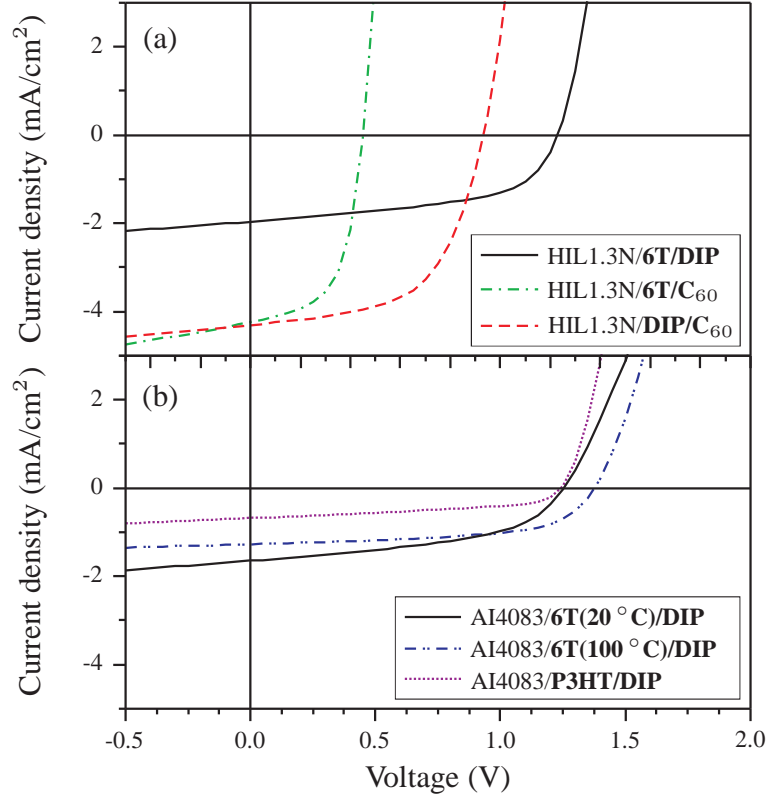

Figure 2 Current-voltage characteristics of the solar cell devices under white LED illumination. (a) Comparison of different D/A systems. (b) Electron accepting performance of DIP when combined with different donors.

band between 500 and $700 \mathrm{~nm}$ and an illumination intensity of $54 \mathrm{~mW} / \mathrm{cm}^{2}{ }^{1}$

The resulting characteristics are shown in Figure 2(a) and important values are summarized in Table 1 . It is evident that all three D/A systems yield proper diodes with fill factors of $54 \%$ for 6T/DIP and $57 \%$ for both $\mathrm{C}_{60}$ solar cells. The open circuit voltage shows a behavior close to the prediction from the intermolecular gaps, where the $V_{\mathrm{oc}}$ of $\mathrm{DIP} / \mathrm{C}_{60}$ approximately doubles the value reached by $6 \mathrm{~T} / \mathrm{C}_{60}$. The $V_{\text {oc }}$ featured by $6 \mathrm{~T} / \mathrm{DIP}$, however, is only 2.7 times that of $6 \mathrm{~T} / \mathrm{C}_{60}$ and thus slightly less than expected. This reduction can possibly be attributed to increased recombination losses in the 6T/DIP solar cells [10]. Indeed,

\footnotetext{
${ }^{1}$ By comparing to $\mathrm{P} 3 \mathrm{HT} / \mathrm{PCBM}$ cells measured under AM1.5g conditions we estimate the LED illumination conditions to correspond roughly to 1 sun for the given cells. Please note that because of these deviations from standard illumination conditions power conversion efficiencies are not specified here.
}

Table 1 Characteristic values of the presented devices illuminated by a white LED at an intensity of $54 \mathrm{~mW} / \mathrm{cm}^{2}$.

\begin{tabular}{lcccc}
\hline Active layer & $\mathrm{HIL}$ & $V_{\mathrm{oc}}(\mathrm{V})$ & $j_{\mathrm{sc}}\left(\mathrm{mA} / \mathrm{cm}^{2}\right)$ & $\mathrm{FF}(\%)$ \\
\hline $6 \mathrm{~T} / \mathrm{C}_{60}$ & $1.3 \mathrm{~N}$ & 0.45 & 4.24 & 56.9 \\
$\mathrm{DIP} / \mathrm{C}_{60}$ & $1.3 \mathrm{~N}$ & 0.93 & 4.31 & 57.0 \\
$6 \mathrm{~T} / \mathrm{DIP}$ & $1.3 \mathrm{~N}$ & 1.23 & 1.97 & 54.0 \\
\hline $6 \mathrm{~T} / \mathrm{DIP}$ & $\mathrm{AI} 4083$ & 1.25 & 1.65 & 48.3 \\
$6 \mathrm{~T}\left(100^{\circ} \mathrm{C}\right) / \mathrm{DIP}$ & $\mathrm{AI} 4083$ & 1.38 & 1.28 & 59.1 \\
$\mathrm{P} 3 \mathrm{HT} / \mathrm{DIP}$ & $\mathrm{AI} 4083$ & 1.24 & 0.69 & 48.0 \\
\hline
\end{tabular}


significantly larger charge recombination rates have been predicted for the D/A pair 6T/perylenediimide (PDI) compared to $6 \mathrm{~T} / \mathrm{C}_{60}$ [11]. Still, keep in mind that varying data for the energy levels of organic semiconductors are found in the literature and the exact energetic landscapes as present in the $6 \mathrm{~T} / \mathrm{C}_{60}$ and $6 \mathrm{~T} / \mathrm{DIP}$ devices are currently being investigated in order to support this conclusion [7]. Both $\mathrm{C}_{60}$ solar cells yield practically identical short circuit currents. This indicates that a major part of the photogenerated charges is contributed by the acceptor in these devices, as confirmed by optical simulations (not shown here). This also explains the reduced short circuit current in the 6T/DIP device due to the lower absorption of DIP compared to $\mathrm{C}_{60}$ as acceptor (Figure 3 ).

To further analyze the electron accepting properties of DIP, the donor layer of the devices was modified (Figure 2(b) and Table 1). 6T grown at room temperature was compared to high temperature growth and poly(3-hexylthiophene) (P3HT, Sepiolid P200, BASF), the most widely studied donor polymer in organic photovoltaics, was combined with DIP as acceptor in a planar heterojunction. In these devices we used the standard PEDOT:PSS formulation AI4083 since there is no need for the high work function of HIL1.3N.

Accompanied by a considerable enhancement of the fill factor, the $V_{\text {oc }}$ of 6T/DIP can be increased to almost $1.4 \mathrm{~V}$ if the substrate is heated to a temperature of $100{ }^{\circ} \mathrm{C}$ during evaporation of the $6 \mathrm{~T}$ film. Note that this voltage is approximately three times the $V_{\text {oc }}$ observed for the $6 \mathrm{~T} / \mathrm{C}_{60} \mathrm{de}$ vice, which implies a reduction of the recombination losses suspected for the room temperature devices. This suppression of recombination might be assisted by orientational effects at the D/A interface induced by a structural change of the $6 \mathrm{~T}$ growth. Orientation dependent recombination rates have been revealed by calculation for the case of 6T/PDI [11]. Improved structural ordering of $6 \mathrm{~T}$ by substrate heating has been reported in the literature [12] and validated by scanning force microscopy and X-ray diffraction (data not shown). Furthermore reduced absorption of the heated film (cf. Figure 3) hints at an unfavourable orientation of the transition dipole moment caused by upright standing molecules [13]. Additionally this lowered absorption is responsible for the decrease in short circuit current observed for this device.

In the last device, $6 \mathrm{~T}$ was replaced by approximately $80 \mathrm{~nm}$ of P3HT spin cast from chloroform and annealed at $75^{\circ} \mathrm{C}$ for 2 hours, essentially switching from a highly crystalline oligothiophene to a largely amorphous polymer (dotted line in Figure 2(b)). Assuming a HOMO level of $4.6 \mathrm{eV}$ [14] for P3HT an intermolecular gap of $1.75 \mathrm{eV}$ is estimated for P3HT/DIP. In agreement with the open circuit voltage, this gap is similar to the 6T/DIP system. Considering the optical gap $E_{\mathrm{g}}=1.9 \mathrm{eV}$ of pristine P3HT, the open circuit voltage of $1.24 \mathrm{~V}$ presented here (as compared to only around $0.5 \mathrm{~V}$ for planar heterojunction $\mathrm{P} 3 \mathrm{HT} / \mathrm{C}_{60}$ cells) closely approaches the limit of this material $[2,15]$.

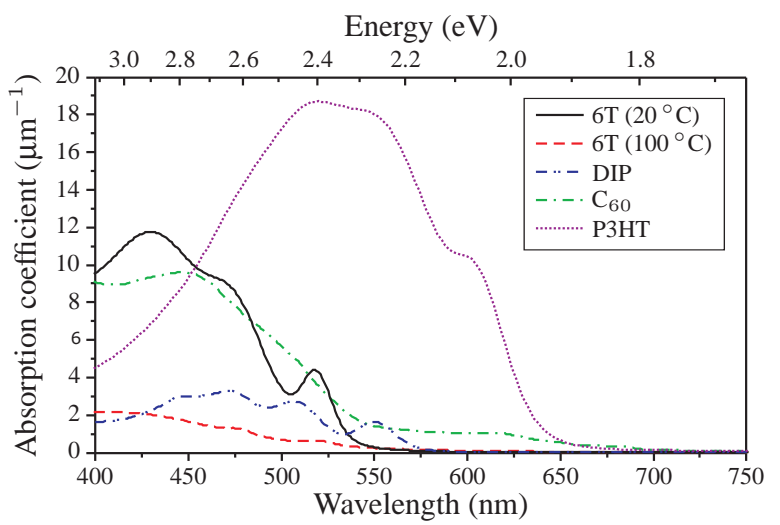

Figure 3 Absorption spectra of the applied organic semiconductors. $\mathrm{C}_{60}$ and P3HT are taken from [17].

Nevertheless, in spite of increased absorption the short circuit current of this device is significantly below the 6T values. This can be ascribed to an exciton diffusion length of only $8.5 \mathrm{~nm}$ in P3HT [16], which is far below the film thickness. Thus a major fraction of excitons is lost before reaching the D/A interface.

In conclusion, diindenoperylene was introduced as a novel acceptor in organic photovoltaic cells. Its applicability was demonstrated for molecular solar cells incorporating the oligothiophene $6 \mathrm{~T}$ as the donor, as well as in a planar heterojunction with the de facto standard solar cell polymer P3HT. For both systems efficient exciton dissociation and charge generation is achieved, while charge recombination is suppressed, manifested by open circuit voltages approaching the ultimate limit.

Acknowledgements We acknowledge the German Research Foundation (DFG) for funding within the priority program SPP1355 and Heraeus Clevios for supplying HIL1.3N. U.H. acknowledges the Bavarian Research Foundation for financial support.

\section{References}

[1] M. Scharber et al., Adv. Mater. 18, 789 (2006).

[2] D. Veldman et al., Adv. Funct. Mater. 19, 1939 (2009).

[3] K. Vandewal et al., Phys. Rev. B 81, 125204 (2010).

[4] C. W. Tang, Appl. Phys. Lett. 48, 183 (1986).

[5] J. Wagner et al., Adv. Funct. Mater. 20, 4295 (2010).

[6] M. Horlet et al., Appl. Phys. Lett. (accepted) (2011).

[7] A. Wilke and N. Koch (HU Berlin), Unpublished.

[8] B. P. Rand et al., Adv. Mater. 17, 2714 (2005).

[9] P. I. Djurovich et al., Org. Electron. 10, 515 (2009).

[10] T. Kirchartz et al., Phys. Rev. B 78, 235320 (2008).

[11] Y. Yi et al., J. Mater. Chem. 21, 1479 (2011).

[12] C. Aruta et al., Appl. Phys. A 97, 387 (2009).

[13] A. Yassar et al., J. Phys. Chem. 99, 9155 (1995).

[14] W. Osikowicz et al., Adv. Mater. 19, 4213 (2007).

[15] J. C. Bijleveld et al., Appl. Phys. Lett. 97, 073304 (2010).

[16] P.E. Shaw et al., Adv. Mater. 20, 3516 (2008).

[17] FLUXiM Setfos (evaluation version) - www.fluxim.com. 